This item was submitted to Loughborough's Research Repository by the author.

Items in Figshare are protected by copyright, with all rights reserved, unless otherwise indicated.

\title{
Anti-inflammatory response to acute exercise is related with intensity and physical fitness
}

PLEASE CITE THE PUBLISHED VERSION

https://doi.org/10.1002/jcb.27810

\section{PUBLISHER}

(c) Wiley

VERSION

AM (Accepted Manuscript)

\section{PUBLISHER STATEMENT}

This is the pre-peer reviewed version of the following article: ATUNES, B.M. ... et al., 2019. Anti-inflammatory response to acute exercise is related with intensity and physical fitness. Journal of Cellular Biochemistry, 120(4), pp. 5333-5342., which has been published in final form at https://doi.org/10.1002/jcb.27810. This article may be used for non-commercial purposes in accordance with Wiley Terms and Conditions for Use of SelfArchived Versions

\section{LICENCE}

CC BY-NC-ND 4.0

\section{REPOSITORY RECORD}

Atunes, Barbara Moura, Eduardo Zapaterra Campos, Ronaldo Vagner Thomatieli dos Santos, Jose Cesar Rosa-Neto, Emerson Franchini, Nicolette Bishop, and Fabio S. Lira. 2019. "Anti-inflammatory Response to Acute Exercise Is Related with Intensity and Physical Fitness”. figshare. https://hdl.handle.net/2134/35057. 


\section{Anti-inflammatory response to acute exercise is related with intensity and physical fitness}

Barbara Moura Antunes ${ }^{1}$, Eduardo Zapaterra Campos ${ }^{1,2}$, Ronaldo Vagner Thomatieli dos Santos $^{3}$, José Cesar Rosa-Neto ${ }^{4}$, Emerson Franchini ${ }^{5}$, Nicolette C Bishop ${ }^{6}$ and Fábio Santos

$$
\text { Lira }^{1}
$$

${ }^{1}$ Exercise and Immunometabolism Research Group, Department of Physical Education, Universidade Estadual Paulista (UNESP), Presidente Prudente, SP, Brazil.

${ }^{2}$ Department of Physical Education, Federal University of Pernambuco, Recife, Brazil.

${ }^{3}$ Department of Bioscience, Universidade Federal de São Paulo (UNIFESP), Santos, SP, Brazil.

${ }^{4}$ Immunometabolism Research Group, Institute of Biomedical Sciences, University of São Paulo (USP), São Paulo, SP, Brazil.

${ }^{5}$ School of Physical Education and Sport, University of São Paulo, São Paulo, Brazil.

${ }^{6}$ School of Sport, Exercise and Health Sciences, Loughborough University, Loughborough, United Kingdom.

Running title: Anti-inflammatory response to acute exercise sessions

\section{Corresponding author:}

Fábio Santos Lira

Exercise and Immunometabolism Research Group, Department of Physical Education, Universidade Estadual Paulista (UNESP), 19060-900, Presidente Prudente, Brazil.

Tel: 55183229 5826; Fax: 551832295388

E-mail: Fabio.lira@unesp.br

Acknowledgments: Barbara Moura Antunes thanks São Paulo Research Foundation (FAPESP) for their support (n²014/08003-1); Fabio Santos Lira thanks National Counsel of Technological and Scientific Development (CNPq) for their support (305263/2015-3 and 444365/2014-1) and Eduardo Zapaterra Campos thanks National Counsel of Technological and Scientific Development (CNPq) for their support (nº401676/2014-15). 


\section{ABSTRACT}

Purpose: The relationship between inflammatory markers and energetic metabolism has been explored. However, the relation between exercise intensity and fitness-status is unclear and it is necessary to understand this relationship to apply specific exercise guidance. The purpose of the study was to analyze metabolic and inflammatory responses imposed by acute exercise sessions performed at moderate, heavy and severe intensities and their relationship with physical fitnessstatus. Methods: Nineteen healthy male volunteers performed three acute exercise sessions until exhaustion or up to 60 minutes on a cycle ergometer at moderate (90\% of VT1), heavy (midpoint between VT1/VT2), and severe (midpoint between VT2/Wmax) intensities. Blood lactate, glucose, NEFA, endotoxin and cytokines were determined for each exercise session. Peripheral and LPS-stimulated release of TNF- $\alpha$, IL-6 and IL-10 were analyzed pre, post and 60-min after sessions. Results: In peripheral blood, severe intensity increased lactate, endotoxin and TNF- $\alpha$ immediately post-exercise and glucose at 60-min post-exercise. There was a trend for IL-10 increase at 60-min post-exercise in peripheral blood. Immediately post-exercise, LPS-stimulated TNF- $\alpha$, IL-6, IL-6/IL-10 ratio and lactate levels were higher in the severe intensity while NEFA levels decreased at this time. At 60-min post-exercise higher concentrations of glucose and a trend for increased IL-10 were observed in severe intensity. Positive correlation was observed between maximal aerobic power and IL-10 ( $\mathrm{r}=0.513, \mathrm{p}=0.042)$ and negative correlations between maximal aerobic power and endotoxin $(\mathrm{r}=-0.531, \mathrm{p}=0.034)$ and lactate $(\mathrm{r}=-0.538$, $\mathrm{p}=0.031$ ) in heavy intensity. Conclusion: Our data show a novel finding that higher cytokine responses occur at higher intensities, mainly in severe intensity. However, the anti-inflammatory (IL-10) response was physical fitness-dependent.

Keywords: Inflammation, Physical fitness status, Exercise intensity, Metabolism, Health. 


\section{INTRODUCTION}

Sedentary lifestyle is a trigger to develop several diseases characterized by low-grade inflammation, including obesity, diabetes mellitus type 2, cardiovascular diseases, and others (Grossmann et al., 2015; Schmidt et al., 2015), showing an increase in 2- to 4-fold in cytokine concentrations (Candore, Caruso, \& Colonna-Romano, 2010), with higher concentrations of Creactive protein, plasminogen activator inhibition-1 (PAI-1) tumor necrosis factor alpha (TNF$\alpha$ ), interleukin 6 (IL-6), and lower concentrations of interleukin 10 (IL-10) and adiponectin (Baker, Hayden, \& Ghosh, 2011; Lira et al., 2010). Cytokines and endotoxin are triggers to proinflammatory pathway activation by interaction with membrane receptors, mainly toll-like receptors, in leucocytes thereby increasing the inflammatory response (Ma \& Suzuki, 2018; Pahl, 1999; Serrano-Marco et al., 2012).

On the other hand, it is clear in the literature the positive effects of exercise training as a nonpharmacological strategy to control low-grade inflammation and endotoxin levels. Evidence shows that trained individuals exhibit lower endotoxemia when compared to sedentary, which is positively correlated with inflammatory markers (PAI-1) in sedentary groups (Lira et al., 2010).

The muscle contraction, mediated by exercise training, produces and releases myokines, especially IL-6 (Pedersen, 2011; Pedersen, Steensberg, \& Schjerling, 2001), that can increase up to 100-fold post-exercise (Starkie, Rolland, Angus, Anderson, \& Febbraio, 2001; Suzuki et al., 2000). IL-6 acts as a precursor for the release of other typically anti-inflammatory cytokines, showing a central role in the cascade activation of IL-10 and IL-1ra (Steensberg, Fischer, Keller, Moller, \& Pedersen, 2003; Suzuki et al., 2003). Besides the anti-inflammatory action, IL-6 acts as an energetic sensor or "energy recruiter” in response to decreased glycogen stores increasing the bioavailability of the energy substrate by glucose metabolism regulation via PI3-K/AKT and GLUT-4 activity and lipolysis stimulation (Reihmane \& Dela, 2014; van Hall et al., 2003).

However, although the relationship between the cytokine associated inflammatory response and energy metabolism has already been explored, it is unclear if this relationship can be 
affected by different exercise intensities and fitness level in healthy men, and if this in turn alters subsequent anti-inflammatory responses. In this line, we hypothesized that there is better effort intensity in physical exercise, due to physical fitness, for the imposition of an anti-inflammatory profile and that such variables (intensity and fitness) should be considered in the daily periodization for health improvement in several groups with different physical fitness level.

Therefore the purpose of the present study was 1) to analyze the effects mediated by acute exercise sessions performed at different intensities on inflammatory and metabolic responses; 2) to investigate the relationship between physical fitness status and immunometabolic profile in healthy men.

\section{METHODS}

Participants: A sample of 19 healthy male volunteers (age $=30 \pm 5$ years; body mass $=74.8 \pm 10.4$ $\mathrm{kg}$; $\mathrm{BMI}=24.2 \pm 2.6 \mathrm{~kg} / \mathrm{m}^{2}$ ) was recruited to participate in this study. The inclusion criteria were: I) Healthy men, II) no health problems preventing high-intensity efforts, and III) no smoking, alcohol intake or illicit drugs. All volunteers were informed about the study protocol procedures, and the study was conducted after signing the Consent form according to the local Ethics Committee (CAAE: 31168714.6.0000.5402).

Experimental Design: On different days, four acute exercise sessions were performed in the same laboratory under controlled conditions (mean temperature $=22.1^{\circ} \mathrm{C}$; mean relative humidity: 55\%; mean barometric pressure: $731.3 \mathrm{mmHg}$ ) by specialized professionals between 8:00 am and 12:00 pm. In the first session, anthropometric and body composition measurements were assessed, and the participants performed a maximal incremental test to exhaustion to determine physical fitness and performance predictors, which was used to prescribe exercise in different intensities. After the maximal incremental test, the other three sessions were performed on alternate days (with at least 48 hours interval between the sessions), at different intensities 
(moderate, heavy and severe) to exhaustion or up to 60 minutes of exercise, in random order and at the same time (8:00 a.m. - 12:00 a.m.). Blood samples were collected pre-exercise, postexercise (immediately after) and 60-min after each session to analyze inflammatory parameters. All participants were instructed not to exercise exhaustively the day before the sessions, not to consume alcohol, caffeine and/or stimulants of any kind during the 24 hours before the tests. Additionally, they were also instructed not to eat a meal less than two hours before the tests. The experimental design is illustrated in figure $\mathbf{1}$.

\section{** Insert figure $1 * *$}

Exercise Protocol: Participants were submitted to a maximal incremental test in a cycle ergometer (Inbrasport CG-04, Embramed, Porto Alegre, Brazil) consisting of 3-minute stages until exhaustion. The fitness condition (sedentary, physically active and well-trained) was initially classified by the International Physical Activity Questionnaire (IPAQ) to determine the initial workload of incremental test and, subsequently, confirmed by aerobic power and maximal oxygen uptake. The initial workload was 35 watts for the untrained individuals, 70 watts for the physically active individuals, and 105 for the well-trained individuals. In all cases, a 25 watts increment was used at each stage, and cycling cadence was maintained between 70-90 rpm maintained (Caputo \& Denadai, 2008). The oxygen uptake $\left(\mathrm{VO}_{2}\right)$ was assessed by a breath-bybreath gas analyzer (Quark PFT, Cosmed ${ }^{\circledR}$, Rome, Italy). The variables measured during the test were: maximal oxygen uptake $\left(\mathrm{VO}_{2 \max }\right)$, assumed as the highest 30 -sec mean observed during the incremental test; maximal heart rate $\left(\mathrm{HR}_{\max }\right)$ (Polar S810i, Polar Electro Oy ${ }^{\circledR}$, Kempele, Finland); and maximum power output $\left(\mathrm{W}_{\max }\right)$. The exhaustion criteria were confirmed by the following variables: gas exchange ratio $>1.1, \mathrm{HR}_{\max }>90 \%$ of the maximum expected for age and rating of perceived exertion $(\mathrm{RPE})>18$. The ventilatory thresholds were determined by three experienced researchers. The first ventilatory threshold (VT1) was determined by first inflection 
point between ventilation (Ve) and workload, and/or the first increase of oxygen equivalent $\left(\mathrm{VE} \cdot \mathrm{VO}_{2}{ }^{-1}\right)$ vs. workload (Binder et al., 2008). The second ventilatory threshold (VT2) was assessed using the second increase of VE vs. workload, and/or the nonlinear increase of dioxide of carbon equivalent (VE· $\mathrm{VCO}_{2}{ }^{-1}$ ) vs. workload (Binder et al., 2008; Papoti et al., 2017). The aerobic exercise sessions started with 5 minutes of warm-up at $30 \%$ of $\mathrm{W}_{\max }$ for all intensities. Next, the participants performed the exercise protocol to exhaustion or up to 60 minutes of exercise on a cycle ergometer, maintaining 70-90 rpm at moderate domain (MD) (i.e. at 90\% of VT1); heavy domain (HD) (midpoint between VT1 and VT2) or severe domain (SD) (midpoint between VT2 and $\mathrm{W}_{\max }$ ) (Binder et al., 2008).

Blood Samples: Blood samples $(20 \mathrm{ml})$ were collected by peripheral puncture of forearm vein at rest, immediately post-exercise and 60 minutes post-exercise (exercise recovery) by nursing staff. The blood samples $(10 \mathrm{ml})$ were immediately allocated into two $5 \mathrm{ml}$ vacutainer tubes (Becton Dickinson, Juiz de Fora, Brazil) containing EDTA for plasma separation, and into one 5 $\mathrm{ml}$ dry vacutainer tube for serum separation. The tubes were kept under refrigeration until the centrifugation and after 1 hour were centrifuged at $3000 \mathrm{rpm}$ for 15 minutes at $4^{\circ} \mathrm{C}$, and plasma and serum samples were stored at $-20^{\circ} \mathrm{C}$ until analysis.

Metabolic analysis: For lactate concentration analysis 25ul of blood from ear lobe were collected and stored in plastic tubes containing 50 ul of $1 \%$ sodium fluoride; subsequently, the samples were analyzed in the lactate analyzer Yellow Springs 1500 Sport. Glucose analyses were performed by a colorimetric method using commercial kits (Labtest ${ }^{\circledR}$, São Paulo, Brazil). Whole blood stimulation with LPS in vitro: For the stimulation of whole blood we used a similar protocol that Abbasi and colleagues (Abbasi et al., 2013) proposed, and $6 \mathrm{ml}$ of venous blood was collected by peripheral puncture of the forearm vein and distributed into two tubes containing K3-EDTA. One blood tube received stimulation with lipopolysaccharide (LPS) (Escherichia coli, type: 0111: B4; Sigma, St. Louis, MO, USA), with a final concentration of 10 ng/ml, and the other tube (control) received 3ul of phosphate-saline buffer (PBS). Both tubes 
were incubated for one hour at $37^{\circ} \mathrm{C}$, with constant and slow rotation, and immediately after incubation, both tubes were centrifuged at $3000 \mathrm{rpm}$ for 15 minutes at $4^{\circ} \mathrm{C}$ to obtain the plasma. Aliquots were made in plastic tubes and stored at $-80^{\circ} \mathrm{C}$ until analysis.

Blood inflammation measurements: Plasma of stimulated and peripheral blood samples was analyzed to assess TNF- $\alpha$, IL-6, IL-10 at pre-session, post-session and 60 minutes post-exercise (recovery).

Stimulated and peripheral blood samples were analyzed by Enzyme-Linked Immunosorbent Assay (ELISA) using high sensitivity kits (R\&D Systems ${ }^{\circledR}$ a biotechne ${ }^{\circledR}$ brand, Quantikine ${ }^{\circledR}$ ELISA, Inc., Minneapolis, USA) with sensitivities of 15.6 - 1000 pg/mL, 3.13 - 300 pg/mL and 7.8 - 500 pg/mL for TNF- $\alpha$, IL-6 and IL-10 respectively, and variation intra and inter assay (\%) of 1.6 - 4.2 and 3.3 - 6.4 (IL-6), 4.2-5.2 and 4.6-7.4 (TNF- $\alpha$ ) 1.7-5.0 and 5.9-7.5 (IL-10), respectively. Non-ester fatty acid (NEFA) was assessed by a colorimetric method with a commercial kit (Wako, 1-2, doshomachi 3-Chome, Chuo-Ku, Osaka 540-8605, Japan). Endotoxin concentration was analyzed pre and post-session by a chromogenic limulus amoebocyte lysate (LAL) test, which is a quantitative test for Gram-negative bacterial endotoxin (Limulus Amebocyte Lysate (LAL) kit; QCL-1000; Lonza; Lonza Walkersville, Inc. Walkersville, MD 21793), using Lyophilized endotoxin (E. coli origin) to generate a standard curve with the chromogenic LAL test kit according to the manufacturer's instructions. The run was performed at $405-410 \mathrm{~nm}$ in spectrophotometer following the end of the reaction, and the correlation between the absorbance and the endotoxin concentration was linear in the range of 0.1-1.0 EU/ml.

Statistical Analysis: Kolmogorov-Smirnov test was used to determine if the data were normally distributed, and descriptive results were shown as means and standard deviation (SD). Comparison of inflammatory and metabolic parameters after different exercise intensities was performed by 2-way ANOVA (domain vs time), with the post-hoc of Bonferroni, when necessary. The effect size was calculated by the ETA Squared $\left(\mathrm{\eta}^{2}\right)$ and analyzed as proposed by 
Cohen (Cohen, 1992) (small: < 0.2; moderate: $0.2-0.8$, and large: > 0.8). In order to analyze the relative variation of blood variables, the percentage delta $(\Delta \%)$ at different exercise intensities (moderate, heavy and severe intensities) was calculated and analyzed by one-way ANOVA. Pearson correlation was used to verify the relationship between metabolic adaptations, performance and fitness condition according intensity domains. Statistical significance was set at 5\%, and all the analyses were conducted using SPSS, release 22.0 (SPSS Inc., Chicago, IL).

\section{RESULTS}

Individual characteristics and performance characterization at maximal incremental test and in each intensity domain are present in the Table $\mathbf{1 .}$

\section{**Insert table $1 * *$}

The Table 2 presents the inflammatory profile, in absolute concentration, of circulating concentrations and LPS-stimulated release into whole blood in all healthy men. In peripheral blood, TNF- $\alpha$ presented a main effect of domain $\left(\mathrm{F}=4.276, p=0.025 ; \eta^{2}: 0.248\right)$ and time ( $F=9.922, p=0.001 ; \eta^{2}: 0.433$ ), however, post-hoc analysis showed only tendency between severe domain and the other both intensities $(p=0.08)$. For the time, higher concentrations were observed immediately post-exercise than in pre and 60 minutes post-exercise. IL-10 presented an interaction between domain and time $\left(\mathrm{F}=2.745, p=0.038 ; \eta^{2}: 0.174\right)$, however the post-hoc was unable to reveal the location of the differences. For LPS-stimulated whole blood, there was an interaction between domain and time for TNF- $\alpha\left(\mathrm{F}=3.610, p=0.011 ; \eta^{2}: 0.205\right)$, however post-hoc analysis was unable to reveal the location of the differences. There was only a time effect $\left(\mathrm{F}=23.305, p<0.001 ; \eta^{2}: 0.625\right)$ for LPS-stimulated whole blood IL-6 release, with greater values immediately post-exercise. 
Metabolic parameters are presented in the Table 3. Glucose presented an interaction between domain and time $\left(\mathrm{F}=3.991, p=0.026 ; \eta^{2}: 0.222\right)$ with concentrations higher in the severe domain at 60 minutes post-exercise compared with the same time in the moderate and heavy domain trials. There was a main effect of domain for lactate ( $\left.\mathrm{F}=53.769, p<0.001 ; \eta^{2}: 0.818\right)$, with greater values in the severe domain than moderate and heavy domains, and also a main effect of time $\left(\mathrm{F}=36.621, \quad p<0.001 ; \eta^{2}: 0.753\right)$ with higher values post-exercise than pre-exercise. Moreover, lactate also presented an interaction $\left(\mathrm{F}=54.168, p<0.001 ; \eta^{2}: 0.819\right)$ with values in the severe domain higher than those in the moderate and heavy intensities immediately postexercise. An effect of time was observed for endotoxin ( $\left.F=7.959, p<0.014 ; \eta^{2}: 0.362\right)$ - with greater values post-exercise than pre-exercise - and also an interaction of domain vs time $\left(\mathrm{F}=3.369, p=0.049 ; \eta^{2}: 0.194\right)$ with higher endotoxin values post-exercise in the severe domain trial when compared with the moderate domain trial.

\section{**Insert table $3 * *$}

In order to account for individual variances in the responses observed the percentage difference was analyzed, by percentage difference $(\Delta \%)$ in peripheral and LPS-stimulated whole blood. There were no differences observed in peripheral blood. However, significant effects were revealed for LPS-stimulated whole blood; whereby the severe domain resulted in a significant \% increase from pre-post exercise for lactate, $(\mathrm{F}=44.890 ; p<0.001)$, IL-6 ( $\mathrm{F}=4.908$; $p=0.011)$ and IL-6/IL-10 ratio $(\mathrm{F}=6.185 ; p=0.004)$ and a parallel \% decrease for NEFA concentrations $(\mathrm{F}=6.063 ; p=0.004)$ (Figure 2).

\section{**Insert figure $2 * *$}

Figure 3 shows the percentage difference $(\Delta \%)$ from pre- to 60 min post-exercise between the intensity domains for metabolic and inflammatory markers. There were no 
differences observed in LPS-stimulated whole blood. Significant differences were found for glucose only; after 60 minutes of recovery higher concentrations of glucose were observed in the higher domain, $(\mathrm{F}=3.487 ; p=0.038)$. There was a tendency for a similar effect for IL-10 $(\mathrm{F}=2.832 ; p=0.068)$ in peripheral blood.

\section{**Insert figure $3 * *$}

In order to analyze the relationship between blood alterations and intensity domains we conducted correlation analyzes observing significant relations with maximal aerobic power $\left(\mathrm{W}_{\max }\right)$.

In peripheral blood was observed inverse correlation between endotoxin and $\mathrm{W}_{\max }(\mathrm{r}=-$ 0.531; $p=0.034$ ) in heavy domain parallel with significant correlation with IL-10 ( $\mathrm{r}=0.513$; $p=0.042)$ and lactate $(\mathrm{r}=-0.538 ; p=0.031)$ variations (Figure 4). A similar response was observed in severe domain verifying inverse correlation between endotoxin and $\mathrm{W}_{\max }(\mathrm{r}=-0.527 ; p=0.025)$ accompanied by a positive relationship between glucose and $\mathrm{W}_{\max }(\mathrm{r}=0.567 ; p=0.014)$ (data not shown).

Interest, in LPS-stimulated whole blood was observed the same positive relationship between IL-10 variation and $\mathrm{W}_{\max }$ dependent of intensity domains, however in lower intensity (moderate domain) (Figure 5).

\section{**Insert figure 4 and figure $5 * *$}

\section{DISCUSSION}

The present study aimed to analyze the effects of acute exercise sessions performed at different intensities on cytokine responses and relationship between physical fitness status and metabolic profile in healthy men. The main results were 1) acute exercise at severe intensity seems to lead to changes in inflammatory parameters, as TNF- $\alpha$, IL-6 levels and IL-6/IL-10 
ratio, lactate and glucose levels while decreased NEFA levels; 2) Cytokines, mainly IL-10 and metabolic parameters were associated with physical fitness status in peripheral and LPSstimulated whole blood.

The most interesting findings of the present study were the relationship between inflammatory profile and maximal aerobic power. This data suggest a positive modulation in IL10 concentrations during high-intensity domains and an effect dependent of the fitness condition. Peake and colleagues (J. M. Peake, Suzuki, Hordern, et al., 2005) in a study conducted with well-trained male runners performing three different exercise sessions (level treadmill running at $60 \% \mathrm{VO}_{2 \max }$ (moderate-intensity trial) for $60 \mathrm{~min}$; level treadmill running at $85 \% \mathrm{VO}_{2 \max }$ (highintensity trial) for $60 \mathrm{~min}$; downhill treadmill running (-10\% gradient) at $60 \% \mathrm{VO}_{2 \max }$ (downhill running trial) for $45 \mathrm{~min}$ ) observed that the concentrations of IL-1ra and IL-10 were significantly higher after the high-intensity trial than after both the moderate-intensity and downhill running trials. These results suggest that following exercise up to 1 hour duration, exercise intensity appears to have a greater effect on antiinflammatory cytokine production by influence of systemic factors, such as stress hormones (cortisol and norepinephrine) and IL-6, as previously mentioned (Agarwal \& Marshall, 2000; J. Peake et al., 2004; J. M. Peake, Suzuki, Wilson, et al., 2005; Sondergaard, Ostrowski, Ullum, \& Pedersen, 2000).

Besides the influence of performance parameters, as intensity in the release of antiinflammatory cytokines other individual factors may influence the IL-10 production. In this way, Jankord and Jemiolo (Jankord \& Jemiolo, 2004) observed in older men that higher volume of regular physical activity was associated with greater concentrations of IL-10 in very active group suggesting that exercise training may play a vital role in controlling inflammatory markers. These studies strengthen and explain our findings corroborating with our hypothesis that exercise in higher intensities is able to promote positive and benefit responses; however the modulations seems to be directly associated with physical fitness status. Although not the main focus of the 
aforementioned studies, both studies by Peake's and Jankord's were conducted with active and/or well-trained individuals, which strengthen the hypothesis that IL-10 modulation is physical fitness-dependent and highlight the novel finding of the present study.

These findings explain the various actions of inflammatory markers in a healthy population, showing that aerobic training status may modulate cytokine responses according to the physiological need imposed by the stress caused by the exercise. This knowledge is extremely important for all audiences, regardless of the physical fitness status, because the results can guide the training progression to obtain the benefits linked to exercise training such as anti-inflammatory response.

Regarding anti-inflammatory responses, our findings are in agreement, at least in part, with the study conducted by Abbasi and colleagues (Abbasi et al., 2013), investigating the production of cytokines in whole blood cultures in response to endotoxin stimulus in athletes after exhaustive exercise. The authors observed an increase in IL-10, IL-1ra, IL-6, MCP-1 and IL-8 in unstimulated blood; on the other hand, it was observed an increase in IL-8, IL-1ra, and TGF- $\beta 1$ with suppression in TNF- $\alpha$ and IL-6 in stimulated blood. However, IL-6 showed a significant peak after 30 minutes of exercise followed by significant attenuation after 3 hours and 24 hours. A similar response regarding IL-6 concentration was observed in LPS-stimulated blood when performing in the severe intensity domain, showing a peak few minutes after exercise when compared to rest. Additionally, we also identified an interaction domain vs time to IL-10 levels possibly reflecting the trend of higher concentrations post-session in severe intensity.

The anti-inflammatory response associated with exercise training is widely shown in the literature given that the exercise training per se can increase IL-6 levels with the peak at postexercise, mainly in aerobic exercise protocols (Pedersen, 2011; Pedersen et al., 2001). Also, this alteration acts as a trigger to regulate anti-inflammatory cytokines, such as IL-10 and IL-1ra (Steensberg et al., 2003). On the other hand, Van der Poll and colleagues (van der Poll, Barber, 
Coyle, \& Lowry, 1996) verified that in endotoxemic patients with hypercortisolemia immediately before the injection of low-dose LPS elevated the plasmatic concentrations of IL10, independently of other cytokines. This effect was not present when individuals were exposed to hypercortisolemia for an extended period. Furthermore, LPS-stimulation induced an increase in IL-10 concentrations which peaked after two hours.

These results corroborate and explain, at least in part, the findings of the present study in which seems to be benefited by positive metabolic modulation in higher intensities. Although not significant, in peripheral blood, it was possible to verify an increase in IL-6 concentration in all intensities, but mainly in higher intensities, which may explain the IL-10 profile. Another hypothesis to be considered is an anti-inflammatory response independent of IL-6 elevation, mediated by hormones and endotoxin alterations, considering that this group showed an increase of endotoxin response to exercise session that is a stressor able to raise cortisol concentrations.

Our data show an increase in LPS-stimulated whole blood concentrations of IL-6 with severe intensity exercise immediately post-exercise and with a trend for higher IL-10 levels 60 minutes post-exercise. It is important to highlight that the significant increase of IL-10 in this intensity began 60 minutes after the end of the session, suggesting that a more pronounced adjustment may occur in later times (for example, 2 hours) as observed by Van der Poll and colleagues (van der Poll et al., 1996). In agreement, the severe domain also presented a significant increase in the IL-6/IL-10 ratio, representing an interaction between pro-inflammatory and antiinflammatory cytokines. This finding suggests that the elevation of IL-10 is smaller than the elevation of IL-6.

IL-6 production and release depend on several training variables, such as volume, exercise mode and/or intensity, and IL-6 alterations is related with energetic regulation given that this cytokine is able to act as an “energy sensor” (Pedersen \& Febbraio, 2008; Reihmane \& Dela, 2014; van Hall et al., 2003). Therefore, the alteration observed in the IL-6/IL-10 ratio may be related to IL-6 release from contracting muscle fibers, which is proportionate to exercise 
intensity, aiming to keep the energy supply for the maintenance of the performance. This energetic contribution can be strengthened by observing the increase of glucose and lactate concentrations post-exercise in the same intensity, given that lactate levels are known to be influenced by depleted glycogen stores due to a low carbohydrate diet or preceding exhaustive exercise (McLellan \& Gass, 1989; Reilly \& Woodbridge, 1999; Yoshida, 1984).

Taking into account the energetic contribution of the glycolytic and oxidative pathways to exercise is important to highlight that there is a reference standard regarding exercise intensity and duration for the uptake and depletion of energy substrates (Ahlborg, Felig, Hagenfeldt, Hendler, \& Wahren, 1974). Exercise performed at low-intensity (25\% to 65\% $\mathrm{VO}_{2 \max }$ ) increases the free fatty acids (FFA) oxidation due to the high energetic demand of the active muscles and the FFA availability from lipolysis (Wolfe, Klein, Carraro, \& Weber, 1990); in turn, exercises performed in high-intensity $\left(>80 \% \mathrm{VO}_{2 \max }\right.$ ) increase the circulation glycolytic flux, plasma lactate concentration, which may reduce the lipolysis and increase re-esterification of fatty acids in the adipose tissue interfering in the regulation of fatty acids mobilization (Wahrenberg, Engfeldt, Bolinder, \& Arner, 1987). Note that is higher the energy expenditure in high intensity exercises, even if the predominant source is not lipids, generating a quantitative increase in the use of lipids since the need for energy demand is higher due to the greater total energy expenditure (Romijn et al., 1993). These explanations justify the NEFA concentration in the severe domain accompanied by lactate and glucose levels elucidating a positive metabolic regulation due to the effort energy demand.

Despite the significant results presented in this study, some limitations were identified and should be considered when interpreting the results. The main limitation is the lack of analysis of other parameters, such as IL-1ra, IL-8, MCP-1, cortisol which undoubtedly would help to enhance the interpretation of the findings and the characterization of the metabolism involved in each exercise intensity domain according to the aerobic fitness-condition. Also, it would be interesting to conduct further studies with the analysis of time-course with more points 
after exercise to confirm the IL-10 profile, mainly in LPS-stimulated whole blood. Other general limitation may be highlighted such as specific population, making necessary studies with other groups, as well as the response in other training protocols.

Taken together, our data show that physiological responses related to cytokine production imposed by several exercise intensities (moderate, heavy and severe) occur due to the activation of the immunological system and higher intensities, mainly the exercise performed in severe domain, showing a relation with anti-inflammatory responses. However, this action is physical fitness-dependent given that modulations in plasma cytokine concentration, mainly IL-10 is directly associated with performance during maximum incremental test among healthy men, especially with maximal aerobic power.

Conflict of interest: The authors declared have no conflict of interest. 


\section{REFERENCES}

Abbasi, A., Fehrenbach, E., Hauth, M., Walter, M., Hudemann, J., Wank, V., . . . Northoff, H. (2013). Changes in spontaneous and LPS-induced ex vivo cytokine production and mRNA expression in male and female athletes following prolonged exhaustive exercise. Exerc Immunol Rev, 19, 8-28.

Agarwal, S. K., \& Marshall, G. D., Jr. (2000). Beta-adrenergic modulation of human type1/type-2 cytokine balance. J Allergy Clin Immunol, 105(1 Pt 1), 91-98.

Ahlborg, G., Felig, P., Hagenfeldt, L., Hendler, R., \& Wahren, J. (1974). Substrate turnover during prolonged exercise in man. Splanchnic and leg metabolism of glucose, free fatty acids, and amino acids. J Clin Invest, 53(4), 1080-1090. doi: 10.1172/JCI107645

Baker, R. G., Hayden, M. S., \& Ghosh, S. (2011). NF-kappaB, inflammation, and metabolic disease. Cell Metab, 13(1), 11-22. doi: 10.1016/j.cmet.2010.12.008

Binder, R. K., Wonisch, M., Corra, U., Cohen-Solal, A., Vanhees, L., Saner, H., \& Schmid, J. P. (2008). Methodological approach to the first and second lactate threshold in incremental cardiopulmonary exercise testing. Eur J Cardiovasc Prev Rehabil, 15(6), 726-734. doi: 10.1097/HJR.0b013e328304fed4

Candore, G., Caruso, C., \& Colonna-Romano, G. (2010). Inflammation, genetic background and longevity. Biogerontology, 11(5), 565-573. doi: 10.1007/s10522-010-9286-3

Caputo, F., \& Denadai, B. S. (2008). The highest intensity and the shortest duration permitting attainment of maximal oxygen uptake during cycling: effects of different methods and aerobic fitness level. Eur J Appl Physiol, 103(1), 47-57. doi: 10.1007/s00421-008-0670-5

Cohen, J. (1992). A power primer. Psychol Bull, 112(1), 155-159.

Grossmann, V., Schmitt, V. H., Zeller, T., Panova-Noeva, M., Schulz, A., Laubert-Reh, D., ... . Wild, P. S. (2015). Profile of the Immune and Inflammatory Response in Individuals With Prediabetes and Type 2 Diabetes. Diabetes Care, 38(7), 1356-1364. doi: 10.2337/dc14-3008

Jankord, R., \& Jemiolo, B. (2004). Influence of physical activity on serum IL-6 and IL-10 levels in healthy older men. Med Sci Sports Exerc, 36(6), 960-964.

Lira, F. S., Rosa, J. C., Lima-Silva, A. E., Souza, H. A., Caperuto, E. C., Seelaender, M. C., . . . Santos, R. V. (2010). Sedentary subjects have higher PAI-1 and lipoproteins levels than highly trained athletes. Diabetol Metab Syndr, 2, 7. doi: 10.1186/1758-5996-2-7

Ma, S., Suzuki, K. (2018). Toll-like Receptor 4: Target of Lipotoxicity and Exercise Induced Anti-inflammatory Effect?. Ann Nutr Food Sci, 2(4): 1027

McLellan, T. M., \& Gass, G. C. (1989). The relationship between the ventilation and lactate thresholds following normal, low and high carbohydrate diets. Eur J Appl Physiol Occup Physiol, 58(6), 568-576.

Pahl, H. L. (1999). Activators and target genes of Rel/NF-kappaB transcription factors. Oncogene, 18(49), 6853-6866. doi: 10.1038/sj.onc.1203239

Papoti, M., da Silva, A. S., Kalva-Filho, C. A., Araujo, G. G., Santiago, V., Martins, L. B., .. . Gobatto, C. A. (2017). Tethered Swimming for the Evaluation and Prescription of Resistance Training in Young Swimmers. Int J Sports Med, 38(2), 125-133. doi: 10.1055/s-0042-105017

Peake, J., Wilson, G., Hordern, M., Suzuki, K., Yamaya, K., Nosaka, K., . . . Coombes, J. S. (2004). Changes in neutrophil surface receptor expression, degranulation, and respiratory burst activity after moderate- and high-intensity exercise. J Appl Physiol (1985), 97(2), 612-618. doi: 10.1152/japplphysiol.01331.2003

Peake, J. M., Suzuki, K., Hordern, M., Wilson, G., Nosaka, K., \& Coombes, J. S. (2005). Plasma cytokine changes in relation to exercise intensity and muscle damage. Eur J Appl 
Physiol, 95(5-6), 514-521. doi: 10.1007/s00421-005-0035-2

Peake, J. M., Suzuki, K., Wilson, G., Hordern, M., Nosaka, K., Mackinnon, L., \& Coombes, J. S. (2005). Exercise-induced muscle damage, plasma cytokines, and markers of neutrophil activation. Med Sci Sports Exerc, 37(5), 737-745.

Pedersen, B. K. (2011). Muscles and their myokines. J Exp Biol, 214(Pt 2), 337-346. doi: 10.1242/jeb.048074

Pedersen, B. K., \& Febbraio, M. A. (2008). Muscle as an endocrine organ: focus on musclederived interleukin-6. Physiol Rev, 88(4), 1379-1406. doi: 10.1152/physrev.90100.2007

Pedersen, B. K., Steensberg, A., \& Schjerling, P. (2001). Exercise and interleukin-6. Curr Opin Hematol, 8(3), 137-141.

Reihmane, D., \& Dela, F. (2014). Interleukin-6: possible biological roles during exercise. Eur J Sport Sci, 14(3), 242-250. doi: 10.1080/17461391.2013.776640

Reilly, T., \& Woodbridge, V. (1999). Effects of moderate dietary manipulations on swim performance and on blood lactate-swimming velocity curves. Int J Sports Med, 20(2), 9397. doi: 10.1055/s-2007-971099

Romijn, J. A., Coyle, E. F., Sidossis, L. S., Gastaldelli, A., Horowitz, J. F., Endert, E., \& Wolfe, R. R. (1993). Regulation of endogenous fat and carbohydrate metabolism in relation to exercise intensity and duration. Am $J$ Physiol, 265(3 Pt 1), E380-391. doi: 10.1152/ajpendo.1993.265.3.E380

Schmidt, F. M., Weschenfelder, J., Sander, C., Minkwitz, J., Thormann, J., Chittka, T., . . . Himmerich, H. (2015). Inflammatory cytokines in general and central obesity and modulating effects of physical activity. PLoS One, 10(3), e0121971. doi: 10.1371/journal.pone.0121971

Serrano-Marco, L., Chacon, M. R., Maymo-Masip, E., Barroso, E., Salvado, L., Wabitsch, M., . . . Vazquez-Carrera, M. (2012). TNF-alpha inhibits PPARbeta/delta activity and SIRT1 expression through NF-kappaB in human adipocytes. Biochim Biophys Acta, 1821(9), 1177-1185. doi: 10.1016/j.bbalip.2012.05.006

Sondergaard, S. R., Ostrowski, K., Ullum, H., \& Pedersen, B. K. (2000). Changes in plasma concentrations of interleukin-6 and interleukin-1 receptor antagonists in response to adrenaline infusion in humans. Eur J Appl Physiol, 83(1), 95-98. doi: $10.1007 / \mathrm{s} 004210000257$

Starkie, R. L., Rolland, J., Angus, D. J., Anderson, M. J., \& Febbraio, M. A. (2001). Circulating monocytes are not the source of elevations in plasma IL-6 and TNF-alpha levels after prolonged running. Am J Physiol Cell Physiol, 280(4), C769-774. doi: 10.1152/ajpcell.2001.280.4.C769

Steensberg, A., Fischer, C. P., Keller, C., Moller, K., \& Pedersen, B. K. (2003). IL-6 enhances plasma IL-1ra, IL-10, and cortisol in humans. Am J Physiol Endocrinol Metab, 285(2), E433-437. doi: 10.1152/ajpendo.00074.2003

Suzuki, K., Nakaji, S., Kurakake, S., Totsuka, M., Sato, K., Kuriyama, T., . . . Sugawara, K. (2003). Exhaustive exercise and type-1/type-2 cytokine balance with special focus on interleukin-12 p40/p70. Exerc Immunol Rev, 9, 48-57.

Suzuki, K., Yamada, M., Kurakake, S., Okamura, N., Yamaya, K., Liu, Q., . . . Sugawara, K. (2000). Circulating cytokines and hormones with immunosuppressive but neutrophilpriming potentials rise after endurance exercise in humans. Eur J Appl Physiol, 81(4), 281-287. doi: 10.1007/s004210050044

van der Poll, T., Barber, A. E., Coyle, S. M., \& Lowry, S. F. (1996). Hypercortisolemia increases plasma interleukin-10 concentrations during human endotoxemia--a clinical research center study. J Clin Endocrinol Metab, 81(10), 3604-3606. doi: 10.1210/jcem.81.10.8855809

van Hall, G., Steensberg, A., Sacchetti, M., Fischer, C., Keller, C., Schjerling, P., . . . Pedersen, B. K. (2003). Interleukin-6 stimulates lipolysis and fat oxidation in humans. J Clin 
Endocrinol Metab, 88(7), 3005-3010. doi: 10.1210/jc.2002-021687

Wahrenberg, H., Engfeldt, P., Bolinder, J., \& Arner, P. (1987). Acute adaptation in adrenergic control of lipolysis during physical exercise in humans. Am J Physiol, 253(4 Pt 1), E383390. doi: 10.1152/ajpendo.1987.253.4.E383

Wolfe, R. R., Klein, S., Carraro, F., \& Weber, J. M. (1990). Role of triglyceride-fatty acid cycle in controlling fat metabolism in humans during and after exercise. Am J Physiol, 258(2 Pt 1), E382-389. doi: 10.1152/ajpendo.1990.258.2.E382

Yoshida, T. (1984). Effect of dietary modifications on lactate threshold and onset of blood lactate accumulation during incremental exercise. Eur J Appl Physiol Occup Physiol, 53(3), 200-205.

\section{Figure Legends:}

Figure 1- Experimental study design

Figure 2- Percentage difference $(\Delta \%)$ between pre and post-session on metabolic and inflammatory profile of healthy men. $\left({ }^{*}\right) \mathrm{p}<0.05,\left({ }^{* *}\right) \mathrm{p}<0.01$ and $(* * *) \mathrm{p}<0.001$

Figure 3- Percentage difference $(\Delta \%)$ between pre and 60 minutes post-session, on metabolic and inflammatory profile of healthy men. $\left(^{*}\right) \mathrm{p}<0.05,\left({ }^{* *}\right) \mathrm{p}<0.01,\left({ }^{* * *}\right), \mathrm{p}<0.001$ and \# tendency a difference.

Figure 4- Relationship between metabolic and inflammatory markers and performance according intensity domains by spontaneous blood.

Figure 5- Relationship between IL-10 and performance measurement, in specific intensity domain, by LPS-stimulated whole blood in healthy men 

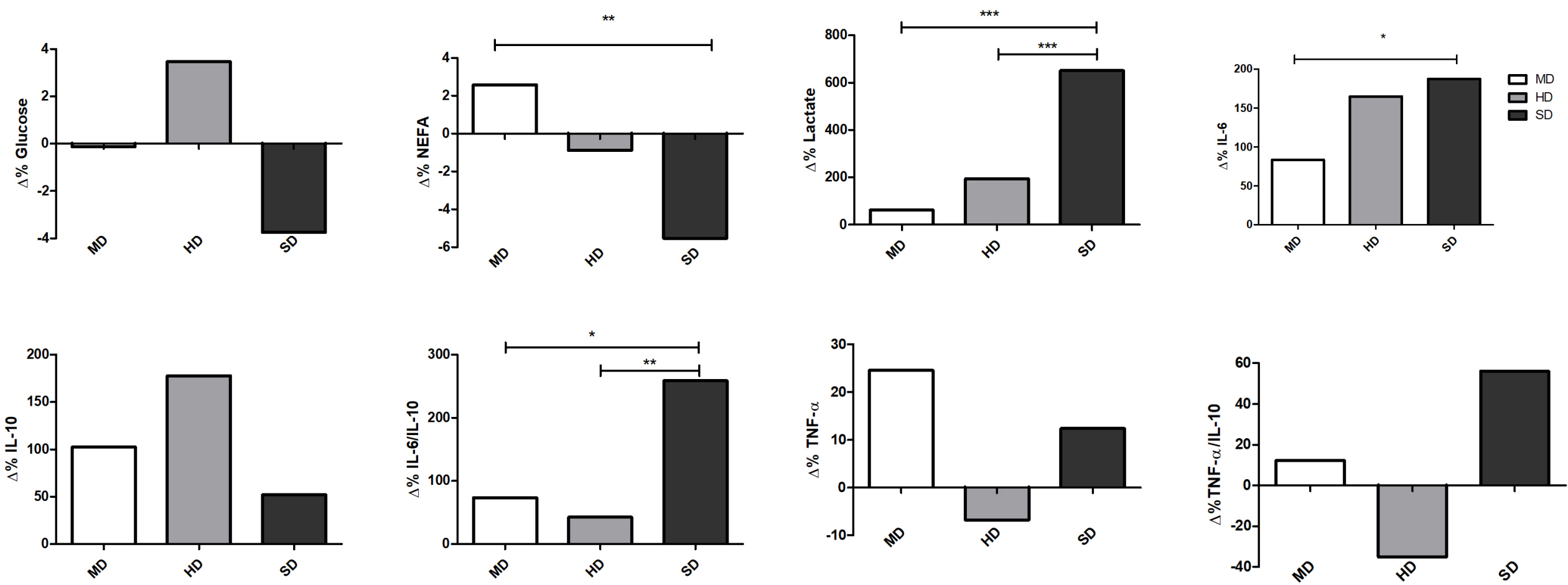

Figure 2: Percentage difference $(\Delta \%)$ between pre and post-session on metabolic and inflammatory profile of healthy men. $\left({ }^{*}\right) p<0.05,\left({ }^{* *}\right) p<0.01$ and $\left({ }^{* * *}\right) p<0.001$ 

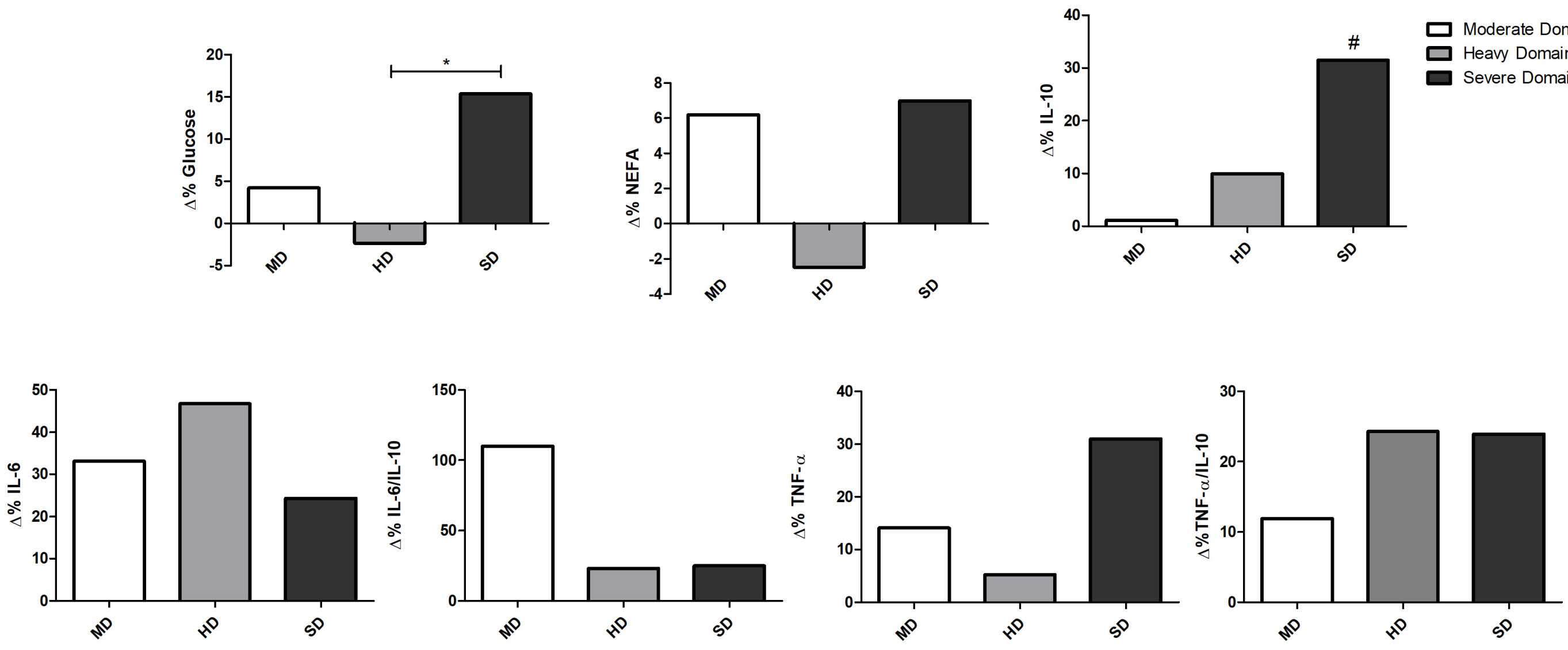

Figure 3: Percentage difference $(\Delta \%)$ between pre and 60 minutes post-session, on metabolic and inflammatory profile of healthy men. $\left({ }^{*}\right) p<0.05,\left({ }^{* *}\right) p<0.01,\left({ }^{* * *}\right)$, $p<0.001$ and \# tendency a difference. 
Table 2. Inflammatory parameters concentration, by peripheral and LPS-stimulated whole blood, in healthy men (data are mean and standard deviation)

\begin{tabular}{|c|c|c|c|c|c|c|c|}
\hline & & \multicolumn{3}{|c|}{ Peripheral blood } & \multicolumn{3}{|c|}{ Stimulated-whole blood } \\
\hline & & Pre & Post & 60-min & Pre & Post & $60-\min$ \\
\hline \multirow{3}{*}{$\begin{array}{l}\text { TNF- } \alpha^{\mathrm{a}, \mathrm{b}, \mathrm{f}} \\
(\text { pg.mL) }\end{array}$} & $M D$ & $2.2 \pm 0.9$ & $2.3 \pm 1.1$ & $2.2 \pm 1.1$ & $386 \pm 196$ & $477 \pm 285$ & $402 \pm 266$ \\
\hline & $H D$ & $2.3 \pm 1.2$ & $3.1 \pm 1.3$ & $2.2 \pm 1.7$ & $435 \pm 296$ & $360.8 \pm 194$ & $369 \pm 199$ \\
\hline & $S D$ & $2.6 \pm 1.5$ & $3.5 \pm 1.8$ & $2.7 \pm 1.2$ & $337 \pm 171$ & $347.6 \pm 197$ & $399 \pm 216$ \\
\hline \multirow{3}{*}{$\begin{array}{c}\text { IL-6 }{ }^{\mathrm{e}} \\
\text { (pg.mL) }\end{array}$} & $M D$ & $1.8 \pm 2.0$ & $2.5 \pm 1.4$ & $2.5 \pm 1.9$ & $9.4 \pm 11.0$ & $15.4 \pm 15.1$ & $9.7 \pm 7.9$ \\
\hline & $H D$ & $1.6 \pm 1.2$ & $3.5 \pm 2.9$ & $2.2 \pm 1.5$ & $10.9 \pm 11.7$ & $23.6 \pm 20.5$ & $12.3 \pm 10.1$ \\
\hline & $S D$ & $1.6 \pm 1.3$ & $2.2 \pm 1.6$ & $2.3 \pm 1.6$ & $8.3 \pm 5.4$ & $21.8 \pm 11.9$ & $9.4 \pm 5.4$ \\
\hline \multirow{3}{*}{$\begin{array}{c}\text { IL-10' } \\
\text { (pg.mL) }\end{array}$} & $M D$ & $3.7 \pm 1.5$ & $3.7 \pm 1.6$ & $3.3 \pm 0.8$ & $7.0 \pm 7.7$ & $11.2 \pm 14.9$ & $7.6 \pm 8.5$ \\
\hline & $H D$ & $3.4 \pm 1.3$ & $3.9 \pm 1.6$ & $3.6 \pm 1.4$ & $6.5 \pm 6.4$ & $18.4 \pm 25.5$ & $9.8 \pm 11.5$ \\
\hline & $S D$ & $3.1 \pm 1.5$ & $3.5 \pm 1.4$ & $3.9 \pm 2.0$ & $7.3 \pm 7.6$ & $4.6 \pm 3.0$ & $7.1 \pm 5.5$ \\
\hline \multirow{3}{*}{$\begin{array}{l}\text { IL-6/IL-10 } \\
\text { ratio }\end{array}$} & $M D$ & $0.6 \pm 0.7$ & $0.7 \pm 0.5$ & $0.8 \pm 0.6$ & $2.9 \pm 4.2$ & $2.2 \pm 1.3$ & $10.0 \pm 29.4$ \\
\hline & $H D$ & $0.6 \pm 0.5$ & $0.9 \pm 0.6$ & $0.6 \pm 0.3$ & $3.3 \pm 3.2$ & $2.5 \pm 2.5$ & $3.7 \pm 6.1$ \\
\hline & $S D$ & $0.5 \pm 0.4$ & $0.7 \pm 0.6$ & $0.7 \pm 0.5$ & $6.7 \pm 15.3$ & $9.8 \pm 11.2$ & $2.6 \pm 2.6$ \\
\hline \multirow{3}{*}{$\begin{array}{l}\text { TNF- } \alpha / \mathrm{IL}-10 \\
\text { ratio }\end{array}$} & $M D$ & $0.6 \pm 0.3$ & $0.7 \pm 0.4$ & $0.7 \pm 0.3$ & $107.8 \pm 163.5$ & $77.9 \pm 59.0$ & $136.4 \pm 199.8$ \\
\hline & $H D$ & $0.7 \pm 0.3$ & $0.9 \pm 0.7$ & $0.7 \pm 0.6$ & $144.7 \pm 148.6$ & $50.7 \pm 52.9$ & $99.8 \pm 103.0$ \\
\hline & $S D$ & $0.9 \pm 0.7$ & $1.2 \pm 0.7$ & $0.9 \pm 0.6$ & $99.9 \pm 109.7$ & $103.1 \pm 93.0$ & $110.2 \pm 131.4$ \\
\hline
\end{tabular}

MD: moderate domain, HD: heavy domain, SD: severe domain. ${ }^{a}$ Domain effect in peripheral blood, ${ }^{b}$ Time effect in peripheral blood, ${ }^{\mathrm{C}}$ Interaction domain vs time in peripheral blood, ${ }^{\mathrm{d}}$ Domain effect in LPS-stimulated blood, ${ }^{\mathrm{e}}$ Time effect in LPS-stimulated blood, ${ }^{\mathrm{f}}$ Interaction domain vs time in LPS-stimulated blood. 
